\title{
Fatores facilitadores e dificuldades no exercício da vigilância sanitária de farmácias em Salvador-Bahia
}

\author{
Facilitating factors and difficulties in the the implementation \\ of sanitary surveillance of drugstores in Salvador, state of Bahia, Brazil
}

Augusto Amorim Bastos ${ }^{1}$

Ediná Alves Costa ${ }^{2}$

Lia Lusitana Cardozo de Castro ${ }^{3}$

\footnotetext{
${ }^{1}$ Complexo Municipal de

Vigilância à Saúde,

Secretaria de Saúde,

Prefeitura Municipal de

Salvador. Av. Vasco da Gama

4.209, Brotas. 40.285-900

Salvador Bahia.

augustobas@gmail.com

${ }^{2}$ Universidade Federal da

Bahia, Instituto de Saúde

Coletiva

${ }^{3}$ Sociedade Brasileira de

Vigilância de Medicamentos
}

\begin{abstract}
Drugstores are the health establishments where medications are dispensed, which is why sanitary surveillance is justified as a means of intervention. The health department uses various technologies to control risk in drugstores. In this study we used the theory of the process of work in health as a benchmark in order to describe and analyze the strengths and difficulties encountered in sanitary surveillance in drugstores in Salvador. Case study methodological strategy was adopted, the analytical categories being facilitating factors and difficulties inherent to technicians, drugstore, sanitary surveillance service and the social environment. Data were collected through direct observation techniques and semi-structured interviews, and the findings were assessed using content analysis. The body of information was processed and categorized using QSR N VIVO software. It was revealed that the nature of the facilitating factors and difficulties found in surveillance activities of drugstores indicate the need for urgent change within the scope of services and the sanitary culture in the country. It also stresses the possibilities of offering a quality service for the protection of health, in a setting conducive to the development of sanitary surveillance and the process of decentralization of these actions.
\end{abstract}

Key words Sanitary surveillance, Drugstore, Risk
Resumo A farmácia é o estabelecimento de saúde onde são dispensados medicamentos, razão que a justifica como objeto de intervenção da vigilância sanitária. Esta utiliza variadas tecnologias para seu controle de risco. Neste estudo utilizou-se referencial da teoria do processo de trabalho em saúde com o objetivo de descrever e analisar facilidades e dificuldades encontradas no exercício da vigilância sanitária de farmácias em Salvador. Adotou-se a estratégia metodológica de estudo de caso, com categorias analíticas facilidades e dificuldades inerentes aos técnicos, farmácia, serviço de vigilância sanitária e ao meio social. Dados foram coletados por meio das técnicas de observação direta e entrevistas semiestruturadas. As falas foram tratadas por meio da análise de conteúdo. O corpus foi processado e categorizado utilizando-se o software QSR N VIVO. Depreende-se que a natureza das facilidades e dificuldades encontradas na realização das atividades de vigilância sanitária de farmácias indicam a necessidade de mudanças emergenciais no âmbito dos serviços e na cultura sanitária do país. Também aponta para a possibilidade de oferta de um serviço de qualidade na proteção da saúde, num cenário favorável ao desenvolvimento da vigilância sanitária como processo de descentralização destas ações. Palavras-chave Vigilância sanitária, Farmácia, Risco 


\section{Introdução}

O comércio de medicamentos, no Brasil, tem-se caracterizado por forte caráter mercantil, centrado na lógica do lucro ${ }^{1-4}$. A comercialização ocorre praticamente à margem da legislação, sem atenção à exigência de prescrição médica ${ }^{5}$, sob intensa influência das estratégias do marketing comercial que estimulam a população a consumir o medicamento como um bem de consumo comum $^{1-4,6}$. Dado que o medicamento é apresentado como o sentir saudável ou ter saúde, este bem social passa a representar um bem de consumo de necessidade ilimitada, comercializado praticamente sem critérios de uso racional ${ }^{2-4}$.

A crescente necessidade de medicamentos, devido à expansão e características da prática médico-terapêutica e o envelhecimento da população, entre outros elementos, levou à ampliação do mercado consumidor e ao crescimento do segmento produtivo de medicamentos, no Brasil, caracterizado pelo surgimento de grande quantidade e diversidade de especialidades farmacêuticas ${ }^{7}$. Este fato contribui para dificultar o controle sanitário em todo o ciclo do medicamento, pois exige aparato estatal moderno e eficiente para acompanhar a dinâmica do mercado farmacêutico e a complexidade dos avanços tecnológicos no setor.

O contínuo lançamento de novos medicamentos e outras tecnologias médicas no mercado, sob eficientes estratégias de promoção comercial, constitui um desafio para a vigilância sanitária acompanhar os avanços tecnológicos e fazer o controle dos riscos que podem surgir com o consumo de novas tecnologias em saúde ${ }^{8}$.

Significativo processo de expansão também ocorreu com a farmácia, pois a mercantilização dos produtos farmacêuticos, a descaracterização da farmácia como estabelecimento de saúde e a falta de critérios de zoneamento, entre outras causas, permitiram a proliferação desse estabelecimento em todo o país. Essa situação demanda significativa parcela da capacidade operacional dos serviços de vigilância sanitária que têm a atribuição de realizar o controle sanitário do comércio de medicamentos.

O medicamento é um bem de natureza dicotômica: por um lado, é uma tecnologia indispensável ao bem-estar social dado que é o instrumento terapêutico, diagnóstico ou profilático mais utilizado em processos mórbidos. Mas, por outro lado, é considerado bem de consumo e, portanto, está inserido na lógica do lucro9. Mes- mo sendo um bem essencial à saúde, o medicamento encontra-se submetido às regras de mercado, cuja racionalidade é pautada pela dinâmica do processo de acumulação de capital. Esta condição híbrida justifica a regulação do mercado e, ao mesmo tempo dificulta a efetivação das estratégias de controle dos riscos sanitários inerentes à produção, à comercialização e ao consumo do medicamento.

No Brasil, diversos estudos demonstram que as farmácias não cumprem a determinação legal referente aos medicamentos que somente devem ser dispensados mediante apresentação de receita médica ${ }^{10-12}$. Vários autores encontraram em farmácias de diversas cidades brasileiras antibióticos ${ }^{13}$ e outros medicamentos enquadrados nessa exigência, tais como antiinflamatórios esteroidais ${ }^{14,15}$, sendo vendidos para consumidores que se queixavam de sintomas de doenças sexualmente transmissíveis ${ }^{16,17}$, otite média aguda ${ }^{18}$, sintomas de rino-sinusite não complicada ${ }^{18}$, dor de dente ${ }^{19}$, etc. Ademais, revelaram que balconistas também faziam diagnóstico e prescreviam medicamentos inadequados ou de qualidade terapêutica duvidosa, segundo classificação de Capellá e Laporte ${ }^{20,21}$.

Face à importância da farmácia, estabelecimento comercial de interesse para a saúde da população, o estudo dos fatores que facilitam ou dificultam as ações de vigilância sanitária dirigidas ao controle dos riscos do medicamento poderá contribuir com subsídios para a reorganização dos serviços, sobretudo no atual processo de municipalização e descentralização da gestão e reorganização do modelo de atenção à saúde. Neste contexto, o estudo tem o objetivo de identificar, descrever e analisar os fatores facilitadores e as dificuldades no exercício da vigilância sanitária de farmácias, em Salvador-Bahia.

Utilizou-se referencial baseado na teoria do processo de trabalho em saúde ${ }^{22-24}$, a partir dos seus elementos constituintes: o agente do trabalho, o objeto, as tecnologias ${ }^{25,26}$, a organização, o produto e a finalidade do trabalho. Compreende-se que o trabalho em vigilância sanitária representa uma modalidade específica de ação na saúde, salientando-se algumas de suas características particulares: atividade exclusiva do Estado voltada essencialmente ao controle de riscos à saúde, agentes com atributo de poder de polícia administrativa e o objeto do trabalho constituirse um bem essencial que está no mercado, tal como o medicamento ${ }^{27}$. 


\section{Métodos}

Trata-se de um estudo de caso ${ }^{27-29}$, exploratório, que descreve e analisa os fatores facilitadores e as dificuldades encontradas pelo Serviço de Vigilância Sanitária no controle sanitário de farmácias, em Salvador. A pesquisa de campo foi realizada no Nível Central do referido serviço. Os dados foram coletados por meio das técnicas de observação direta e realização de entrevistas semiestruturadas. Os participantes foram informados dos objetivos do estudo, solicitando-se a autorização por meio de um Termo de Consentimento Livre e Esclarecido.

A observação direta ocorreu no período de 28/07/2005 a 13/09/2005, em momentos pré-determinados, quando os técnicos executavam atividades de vigilância sanitária de farmácias.

Contou-se com 21 momentos de observação direta do trabalho dos técnicos sobre as farmácias, com a utilização de um roteiro previamente elaborado. Foram registradas anotações em diário de campo que serviram para complementar, sustentar e qualificar o corpus das entrevistas.

Foram realizadas 17 entrevistas semi-estruturadas com os seguintes participantes: o anterior e o atual coordenador de saúde ambiental, o sub-coordenador de vigilância sanitária, o chefe do setor de serviços e 13 técnicos que executam atividades de vigilância sanitária de farmácias. Estes informantes-chave foram selecionados seguindo os critérios de formação profissional e tempo de serviço em vigilância sanitária de farmácias.

Em relação à formação do agente, conformaram-se cinco grupos: nutricionista, médico veterinário, farmacêutico, engenheiro/arquiteto e outras profissões da saúde. Quanto ao tempo de serviço, foram formados três grupos: 0 a 3 anos, 3 a 10 anos e acima de 10 anos de exercício em vigilância sanitária. O cruzamento desses dados resultou em 13 subgrupos, selecionando-se um informante-chave de cada subgrupo. Procurou-se diversificar os sujeitos em função dos critérios adotados.

Foram excluídos os técnicos lotados nos Serviços de Vigilância Sanitária dos Distritos Sanitários, os cedidos a outros órgãos, os que estavam em licença sem vencimentos, os que ocupavam cargo em comissão não relacionado ao trabalho da vigilância sanitária de farmácias, aqueles que não exerciam atividades de vigilância sanitária de farmácias e os profissionais de nível médio.

Os contatos com os informantes-chave foram feitos diretamente na instituição ou por telefone, ocasião em que se apresentava o projeto de investigação, salientando-se sua natureza acadêmica. Não houve recusas para as entrevistas, tampouco quanto aos momentos de observação direta. As entrevistas, com duração média de 30 minutos, foram realizadas no período de 03/08/ 2005 a 03/09/2005, na Secretaria Municipal de Saúde (SMS), com garantia de privacidade. Uma entrevista foi realizada no Serviço Estadual de Vigilância Sanitária e duas outras nas respectivas residências dos entrevistados, em semelhantes condições de privacidade.

Nas entrevistas foram utilizados roteiros, submetidos a pré-teste com técnicos de um Distrito Sanitário que desenvolviam atividades de vigilância sanitária de farmácias. As entrevistas foram gravadas em fitas magnéticas e transcritas para arquivos no software Word, versão 2000. Fez-se revisão das transcrições por comparação do áudio com o texto transcrito. Os segmentos dos textos oriundos das entrevistas foram classificados de acordo com as categorias analíticas, conforme metodologia preconizada por Emerson et al..$^{30}$. As falas dos entrevistados foram tratadas por meio de análise de conteúdo proposta por Minayo ${ }^{31}$ e Bardin ${ }^{32}$.

O corpus foi processado, organizado e disposto em categorias, nas seguintes unidades de análise: fatores facilitadores e dificuldades encontradas no exercício da vigilância sanitária de farmácias. Foi utilizado o software QSR N VIVO, versão 2.0 que possibilitou a identificação de similitudes e divergências entre as falas dos informantes e as notas de observação direta. O processo de triangulação permitiu a comparação dos dados obtidos das entrevistas com aqueles oriundos da observação direta, o que levou ao desenvolvimento de linhas convergentes de investigação visando à confirmação do mesmo fenôme$\mathrm{no}^{29,30}$. A análise e a interpretação dos dados foram conduzidas a partir da articulação estabelecida com o arcabouço teórico que fundamentou o estudo. Os resultados são apresentados de forma descritiva, por meio de textos digitados no programa Word, versão 2000.

Os fatores facilitadores e as dificuldades na realização do trabalho de vigilância sanitária de farmácias, identificados pelo estudo, foram sistematizados de acordo com sua natureza, nas seguintes categorias analíticas: relativamente aos agentes, ao objeto (farmácia), ao serviço de vigilância sanitária e ao meio social. Esta classificação constituiu um esforço didático para caracterizar distinções, embora tais aspectos sejam inter-relacionados (Quadro 1). 
Quadro 1. Fatores facilitadores e dificuldades no exercício da vigilância sanitária de farmácias, em SalvadorBahia, relacionados aos técnicos, à farmácia, ao serviço e ao meio social

\begin{tabular}{|c|c|c|}
\hline & Fatores facilitadores & Dificuldades \\
\hline Técnicos & $\begin{array}{l}\text { Experiência profissional; } \\
\text { Qualificação. }\end{array}$ & $\begin{array}{l}\text { Insuficiência de profissionais farmacêuticos; } \\
\text { Insuficiência de capacitação técnica; } \\
\text { Inexistência de estratégias de socialização de } \\
\text { saberes; } \\
\text { Acompanhamento da dinâmica do mercado } \\
\text { farmacêutico. }\end{array}$ \\
\hline Farmácia & $\begin{array}{l}\text { Menor quantitativo e maior } \\
\text { organização da farmácia em relação } \\
\text { a outros estabelecimentos sob } \\
\text { vigilância sanitária; } \\
\text { Extensa regulamentação. }\end{array}$ & $\begin{array}{l}\text { Comercialização de produtos e serviços estranhos } \\
\text { ao comércio farmacêutico; } \\
\text { Resistência nos casos de transgressão da legislação } \\
\text { sanitária; } \\
\text { Ausência do farmacêutico na farmácia; } \\
\text { Localização do estabelecimento em áreas de difícil } \\
\text { acesso. }\end{array}$ \\
\hline $\begin{array}{l}\text { Serviço de } \\
\text { Visa } \\
\text { (Vigilância } \\
\text { Sanitária) }\end{array}$ & $\begin{array}{l}\text { Disponibilidade de transporte; } \\
\text { Disponibilidade de formulários } \\
\text { padronizados e computador com } \\
\text { acesso à internet; } \\
\text { Organização do trabalho em equipe; } \\
\text { Priorização das farmácias. }\end{array}$ & $\begin{array}{l}\text { Deficiência de infraestrutura (pequeno espaço } \\
\text { físico, acesso difícil a tecnologias de informação e } \\
\text { insuficiência de recursos humanos); } \\
\text { Falta de padronização de procedimentos; } \\
\text { Inexistência de setor jurídico formalizado. }\end{array}$ \\
\hline Meio social & $\begin{array}{l}\text { Extensa regulamentação sanitária; } \\
\text { Realização de atividades com outros } \\
\text { órgãos e sua divulgação nos meios } \\
\text { de comunicação. }\end{array}$ & $\begin{array}{l}\text { Desconhecimento, por parte da maioria da } \\
\text { população, dos riscos inerentes ao consumo de } \\
\text { medicamentos; } \\
\text { Incipiente controle social da vigilância sanitária; } \\
\text { Falta de informação nas instituições afins à área } \\
\text { da saúde sobre a função da vigilância sanitária; } \\
\text { Concepção da farmácia como estabelecimento } \\
\text { comercial, no Brasil; } \\
\text { Pressão do poder econômico sobre a vigilância } \\
\text { sanitária; } \\
\text { Complexidade da dinâmica do mercado } \\
\text { farmacêutico. }\end{array}$ \\
\hline
\end{tabular}

\section{Resultados e discussão}

O Quadro 1 sistematiza os achados do estudo, provenientes das entrevistas e da observação direta das atividades de vigilância sanitária de farmácias, no tocante aos fatores facilitadores e às dificuldades relacionadas aos agentes do trabalho, à farmácia, ao serviço de vigilância sanitária e ao meio social.

\section{Fatores facilitadores à realização das atividades de vigilância sanitária nas farmácias}

Como elementos facilitadores relacionados aos agentes, na realização das atividades de vigilância sanitária nas farmácias, destacaram-se a experiência profissional e a qualificação para o trabalho. O saber prático foi considerado importante na condução de situações que implicam em medidas enérgicas, tais como interdição de estabelecimento, apreensão de produtos, casos que requerem habilidades para se lidar com conflitos de interesses. Essas habilidades são adquiridas à medida que os técnicos experimentam situações semelhantes. A qualificação para o trabalho é essencial para prover o conhecimento técnico necessário às atividades de controle sanitário e conferir segurança sobre o que está sendo realizado pelos profissionais no próprio ambiente regulado.

Entre os fatores facilitadores relacionados à farmácia enquanto objeto de trabalho da vigi- 
lância sanitária, foram mencionados o menor número relativo de estabelecimentos e a maior organização da farmácia quando comparada com outros objetos sob controle sanitário. Além disso, a extensa regulamentação dos medicamentos e um maior controle conferido à farmácia contribuem para melhorar a adequação deste estabelecimento às normas sanitárias.

A regulamentação do setor farmacêutico constitui um elemento facilitador da execução das ações de vigilância sanitária. A legislação de proteção e defesa da saúde, fundamentada em saberes necessários à função regulatória, estabelece os comportamentos desejados de cada parte, não apenas dos agentes econômicos, mas também dos reguladores ${ }^{3}$. Desse modo, a norma se converte em importante instrumento para orientar as atividades dos técnicos.

O fato da legislação determinar um conjunto de exigências interdependentes para o exercício do comércio farmacêutico obriga a farmácia a cumprir as normas ${ }^{33-37}$. Por exemplo, a farmácia sem licença sanitária não pode adquirir medicamentos dos distribuidores, os quais, por seu lado somente devem fornecê-los para estabelecimentos licenciados pela vigilância sanitária. Esses rearranjos normativos foram estabelecidos mais recentemente, após os muitos eventos de derrame de medicamentos falsificados no mercado, na segunda metade dos anos $1990^{3}$.

O conjunto de fatores facilitadores da realização das atividades de vigilância sanitária nas farmácias, relacionados ao serviço de vigilância sanitária, diz respeito a aspectos da infraestrutura, organização do trabalho e gestão. Os itens transporte, disponibilidade de legislação e determinados instrumentos, tais como formulários (termos), roteiro de inspeção e computador com acesso à rede web foram enfatizados, pelos entrevistados, como condições que facilitam a realização das atividades.

Os depoimentos dos entrevistados e a observação direta de suas atividades revelaram que a organização do trabalho em equipe, principalmente quando das atividades nos estabelecimentos farmacêuticos, permite a troca de opiniões e o compartilhamento de apreciações técnicas que favorecem a tomada de decisão; além disso, contribui para a criação de um ambiente de trabalho onde os técnicos se sentem mais seguros nos diversos momentos do processo de trabalho.

Os técnicos reiteraram que o serviço prioriza as farmácias entre os objetos de intervenção, mas não esclareceram o motivo. Pode-se pensar que é devido à maior visibilidade dos riscos inerentes aos medicamentos, como também porque estas competências foram recentemente descentralizadas para o município de Salvador, despertando este interesse.

Os entrevistados destacaram a importância do apoio político-administrativo por parte da gestão da SMS para o trabalho de vigilância sanitária; este apoio foi considerado fundamental para fortalecer a autonomia do corpo técnico e criar um ambiente de segurança e respeito às decisões tomadas, que muitas vezes são objeto de pressões políticas.

No tocante às facilidades relacionadas ao meio social, os técnicos ressaltaram a regulamentação sanitária minuciosa do setor farmacêutico e a divulgação de atividades de caráter intersetorial, realizadas em determinadas situações. $\mathrm{O}$ fato da farmácia e de toda a cadeia do medicamento ser objeto de extensa regulamentação não apenas facilita como legitima o trabalho da vigilância sanitária. Foi conferido destaque e importância à realização de ações conjuntas com outros órgãos, especialmente o Conselho de Farmácia e o Ministério Público. Considerou-se ainda que a divulgação dessas ações nos meios de comunicação repercute positivamente e facilita a atuação, pois confere maior visibilidade às atividades de vigilância sanitária nas farmácias, perante a população e o segmento produtivo.

\section{Dificuldades na realização das atividades de vigilância sanitária de farmácias}

As principais dificuldades relacionadas aos agentes de vigilância sanitária, obtidas dos depoimentos e da observação direta das atividades, referem-se à composição deficitária das equipes, a insuficiente capacitação técnica e limitações para acompanhar a dinâmica do mercado farmacêutico.

Observou-se a formação de equipes sem inclusão do farmacêutico para a realização de atividades de vigilância sanitária em farmácias. $\mathrm{Na}$ opinião de alguns entrevistados, isto é preocupante, devido ao requisito de conhecimentos técnicos sobre o medicamento para que o profissional tenha condições de realizar um efetivo controle sanitário do estabelecimento farmacêutico.

A problemática da qualificação e capacitação para o trabalho foi um tema recorrente. Os técnicos se ressentem de não haver uma política bem definida de capacitação dos profissionais recémadmitidos no serviço; o que se soma à inexistência de estratégias para socialização de saberes entre eles. Algumas falas ressaltaram que, na realidade, os técnicos mais novos aprendem com os 
profissionais mais experientes, no momento do fazer; e isto foi motivo de preocupação, devido à falta de padronização de procedimentos e variabilidade na execução das atividades, o que não deve ser exemplo para os novos profissionais. A falta de estratégias para a troca de conhecimentos, a exemplo de reuniões técnicas, dificulta a discussão sobre o trabalho desenvolvido.

A formação e a capacitação dos técnicos influenciam na qualidade do trabalho, na credibilidade dos profissionais e do serviço de vigilância sanitária perante as farmácias e a população. Algumas das repercussões desfavoráveis da insuficiente qualificação profissional são a ausência de padrões mais uniformes nas atividades e a insegurança do técnico que pode se revelar na tomada de decisão em situações que exigem conhecimentos para fundamentar as providências adotadas e não apenas uma apropriação da legislação correspondente. A qualificação profissional adequada é crucial para a apreensão do objeto de trabalho e o alcance de sua finalidade; neste caso, a prevenção de agravos à saúde mediante o controle de riscos no estabelecimento farmacêutico.

As principais dificuldades relacionadas ao objeto - a farmácia - dizem respeito à resistência dos seus responsáveis às medidas de vigilância sanitária, à comercialização de produtos e serviços estranhos ao comércio farmacêutico, à ausência do farmacêutico e à localização de farmácias em bairros de difícil acesso.

Foi possível observar que a resistência às medidas de vigilância sanitária costuma ocorrer em casos de transgressões da lei, quando os técnicos encontram situações que representam risco à saúde da população. A aplicação de medidas de natureza coercitiva, como a interdição do estabelecimento e até mesmo a lavratura de Auto de Infração, provoca reações dos responsáveis pelo estabelecimento contra os profissionais, com apelos e argumentos que resultam em intimidações.

As falas de alguns entrevistados e a observação das atividades evidenciaram a descaracterização do estabelecimento farmacêutico, devido à exposição e venda de produtos e serviços não pertinentes à farmácia, tais como alimentos, pagamentos de contas e outros. De acordo com os técnicos, estas atividades resultam de liminares concedidas pela justiça em favor das farmácias.

Alguns entrevistados ressaltaram a ausência do farmacêutico como uma dificuldade para o trabalho de vigilância sanitária. A maioria desses estabelecimentos não tem grande interesse que o profissional permaneça na farmácia, devido ao problema da remuneração. Como não o remu- neram adequadamente, também não exigem o cumprimento da carga horária e sua permanência para realizar atividades de atenção farmacêutica e controle dos medicamentos.

Dificuldade adicional ao controle do comércio farmacêutico, destacada pelos técnicos, foi a problemática decorrente da localização de farmácias em bairros periféricos, em geral de difícil acesso. Tal situação apareceu nos depoimentos dos entrevistados, que manifestaram preocupações quanto à segurança pessoal nessas áreas e relataram a existência destes estabelecimentos funcionando sem licença sanitária.

Estabelecimentos farmacêuticos são instalados cada vez em maior número e em qualquer lugar porque não há um sistema de zoneamento, preconizado pela Organização Mundial de Saúde (OMS). A dificuldade supracitada poderia ser minimizada com a descentralização dos serviços de vigilância sanitária para os Distritos Sanitários, organizados sob a estratégia da territorialização ${ }^{38}$. Isto permitiria identificar e conhecer o que é de interesse da saúde na área de abrangência de cada Distrito, proporcionando a aproximação dos técnicos aos estabelecimentos sujeitos a controle sanitário e seus respectivos problemas ${ }^{38-40}$.

Além dessa problemática, existe variação nas características do estabelecimento farmacêutico, conforme a localização e a clientela atendida, com mudanças no perfil da oferta de medicamentos, em função do nível sócio-econômico predominante no local. De acordo com o extrato social da população há padrões diferenciados de farmácia quanto aos tipos de medicamentos, preços, instalações, serviços oferecidos e estratégias de promoção dos produtos comercializados. Certamente, estas condições influenciam no trabalho realizado pelos técnicos que devem estar atentos às características de cada farmácia.

Quanto às dificuldades relacionadas ao serviço de vigilância sanitária conferiu-se ênfase a deficiências na infraestrutura, falta de padronização de procedimentos e inexistência de setor jurídico formalizado. A deficiência na infraestrutura se reflete em exíguo espaço físico, acesso difícil a tecnologias de informação e insuficiência de recursos humanos, sobretudo profissionais farmacêuticos. Tal situação constitui limitações importantes na realização das atividades, não somente de vigilância sanitária das farmácias, como também de outros estabelecimentos. O número de farmacêuticos lotados no Nível Central da Vigilância Sanitária foi considerado insuficiente face ao grande número de farmácias na cidade de Salvador. 
As limitações na infraestrutura do serviço, segundo os entrevistados, dificultam o atendimento às necessidades atuais. Os técnicos expressaram preocupações com o processo em curso de descentralização das ações de vigilância sanitária do Estado para o município de Salvador, o que implicará em novas responsabilidades. No seu entender, o aumento de atividades, com a manutenção de uma infraestrutura deficiente, poderá repercutir negativamente na qualidade do serviço e levar à não prioridade das farmácias, tal como ocorreu com o comércio varejista de alimentos.

Outra ordem de dificuldades relativas ao serviço decorre da falta de padronização dos procedimentos realizados pelos profissionais. Alguns entrevistados relataram a existência de variação entre um técnico e outro, não somente na realização das atividades, como também nas exigências feitas ao estabelecimento farmacêutico, quando das inspeções sanitárias. Foi possível observar ausência de uniformização das atividades que podem provocar consequências negativas para a qualidade do trabalho executado.

Além dessas dificuldades, foi destacado o fato do serviço não possuir um setor jurídico formalizado, com profissionais do direito para o exercício da função de assessoria jurídica, tão necessária no processo de trabalho da vigilância sanitária. Conta-se apenas com o apoio da Procuradoria quando são feitas solicitações, em situações específicas. Segundo alguns técnicos, isto é insuficiente, pois o cotidiano da vigilância sanitária envolve frequentes questões de natureza jurídica.

No tocante às dificuldades inerentes ao meio social, as principais questões apontadas pelos entrevistados foram: o desconhecimento da população sobre os riscos relacionados aos medicamentos, o tênue controle social na vigilância sanitária, a falta de informação sobre a função desta nas instituições afins ao setor saúde, a concepção vigente no país da farmácia como estabelecimento comercial, a pressão do poder econômico no âmbito do comércio farmacêutico e as limitações para acompanhar a dinâmica desse mercado.

A falta de conhecimento, por parte da maioria da população, acerca dos riscos à saúde que o consumo de medicamentos representa revelouse uma preocupação frequente dos entrevistados. Suas falas manifestavam inquietações quanto a uso inadequado de medicamentos, sem critérios e sem o devido acompanhamento médico e de outros profissionais da saúde.

Esse desconhecimento quanto aos riscos dos medicamentos contribui para o consumo indis- criminado. A maioria da população adquire facilmente os produtos farmacêuticos diretamente nas farmácias, sem prescrição médica, fenômeno bem evidenciado na literatura científi$\mathrm{ca}^{13,14,16,17,19}$. Ademais, a automedicação é estimulada pelas estratégias de promoção e o consumo é induzido por balconistas e proprietários leigos com o objetivo de maximizar as vendas.

Assim como os riscos relacionados aos medicamentos, a vigilância sanitária também ainda não é de fato conhecida na sociedade; pode-se dizer que nem mesmo no ambiente da saúde. Assim, o desconhecimento, por parte de instituições afins, da sua função social constitui uma dificuldade adicional no trabalho de controle sanitário das farmácias. Na maioria das vezes, a percepção das ações de vigilância sanitária limita-se à função fiscalizadora, também porque sua atuação, ainda hoje, quase sempre se reduz a esta atividade, que não é acompanhada de informação para as comunidades onde se localizam as farmácias.

A escassa participação popular no controle social da vigilância sanitária foi destacada por alguns técnicos que entendem que isto pode implicar em dificuldade para o controle sanitário da farmácia. Este problema foi relacionado ao desconhecimento, entre a população, da importância das ações de vigilância sanitária na proteção da saúde; também justificaria a tênue atuação dos atores sociais nas instâncias colegiadas do SUS, no referente às questões da vigilância sanitária no sentido de uma melhor organização e efetiva implementação das ações.

Outra dificuldade do âmbito social referida é a concepção vigente da farmácia como um estabelecimento comercial. Segundo os entrevistados, isto pode também influenciar desfavoravelmente no controle de riscos à saúde no referente ao comércio farmacêutico. A falta de critérios que organizem a distribuição das farmácias no território da cidade leva à proliferação indiscriminada desses estabelecimentos. Além disso, a ampla margem de liberdade de comercialização, semelhante ao comércio comum, desconsidera os riscos inerentes ao medicamento.

Os entrevistados também ressaltaram a pressão do poder econômico que afeta o trabalho da vigilância sanitária: seja pelas demandas para a regularização de suas atividades - para adquirir medicamentos a farmácia necessita comprovar o licenciamento sanitário - seja pela busca de autorização judicial para comercializar produtos considerados pela vigilância sanitária estranhos ao comércio farmacêutico. Alguns profissionais sinalizaram dificuldades em acompanhar 
a dinâmica do mercado farmacêutico; o avanço técnico e científico traz mudanças no setor, o que requer melhor organização e estrutura do serviço de vigilância sanitária, obtenção de informação em tempo hábil e legislação atualizada.

O segmento farmacêutico está em constantes modificações. Acompanhar essa dinâmica representa um desafio aos técnicos devido às constantes inovações terapêuticas e ampliação do mercado $^{41}$. Estes aspectos, aliados à fragilidade na infraestrutura do serviço, são fatores que dificultam a atualização dos profissionais em relação a tais mudanças.

A dinâmica do mercado farmacêutico também provoca mudanças nas farmácias, no Brasil, com ampliação das atividades ali desenvolvidas, a exemplo da venda de produtos considerados estranhos ao comércio farmacêutico, permitida por força de liminar judicial. A vigilância sanitária não consegue conter o poderio econômico das grandes redes que obtêm liminares autorizando-as a comercializar quaisquer produtos. Isto contribui para descaracterizar a farmácia como estabelecimento de saúde ${ }^{17}$. Portanto, dificuldades de natureza operacional e questões de ordem jurídica interferem no processo de trabalho, já por si mesmo complexo por ser exercido em ambiente externo ao serviço; em consequência limitam o cumprimento da função regulatória na proteção da saúde.

A vigilância sanitária ainda não é, de fato, conhecida na sociedade; pode-se dizer que nem mesmo no ambiente da saúde. Desta maneira, o desconhecimento, por parte de instituições afins - a exemplo do Judiciário - da função social da vigilância sanitária dificulta, adicionalmente, o trabalho de controle sanitário das farmácias. Frequentemente, a percepção das ações de vigilância sanitária limita-se à função fiscalizadora; também porque sua atuação, ainda hoje, quase sempre se reduz a esta atividade.

\section{Considerações finais}

A partir do entendimento da atuação em vigilância sanitária como trabalho em saúde de natureza específica, identificou-se, neste estudo, um conjunto de fatores que facilitam e dificultam a organização e o exercício das atividades de vigilância sanitária de farmácias.

As dificuldades que se apresentam para o trabalho de controle sanitário do estabelecimento farmacêutico são de natureza diversificada. A insuficiente qualificação e capacitação dos profis- sionais, a ausência de padrões técnicos nas atividades, a insuficiência da infraestrutura e da organização do serviço, necessários à operacionalização das atividades, se somam às dificuldades relacionadas ao meio social. Estas decorrem principalmente, da concepção da farmácia como estabelecimento tão somente comercial, do desconhecimento da população quanto aos riscos inerentes aos medicamentos, bem como da pouca valorização social da vigilância sanitária para a proteção da saúde.

Do conjunto de dificuldades apresentadas, as referentes ao meio social sem dúvida requerem maior esforço para serem superadas; estão relacionadas a fatores oriundos do mercado, de natureza política e sócio-cultural que influenciam nas atividades regulatórias do comércio farmacêutico. Esta situação relaciona-se historicamente à forma pouco cuidadosa como o medicamento e a farmácia têm sido tratados no Brasil.

Entre os fatores facilitadores da vigilância sanitária de farmácias, destaca-se o conhecimento técnico sobre o objeto de trabalho, o domínio da legislação como instrumento de trabalho, a realização de ações intersetoriais e o apoio político da gestão, indispensável ao enfrentamento de resistências e pressões de ordem econômica. A extensa regulamentação sanitária do segmento farmacêutico constitui um elemento que favorece a realização das atividades porque normatiza o objeto sob controle sanitário em diversos aspectos interdependentes.

O presente trabalho levanta indagações que indicam a necessidade de realização de estudos mais aprofundados sobre o processo de trabalho da vigilância sanitária, suas dificuldades e seus fatores facilitadores no controle sanitário da farmácia. A produção científica na temática da vigilância sanitária no Brasil ainda é relativamente pequena, portanto, muitos dos achados precisam ser cotejados com outros estudos de natureza similar.

Este estudo não permite afirmar que as atividades investigadas alcançam a finalidade de proteção e defesa da saúde; tampouco possibilita fazer generalizações a respeito do modelo tecnológico e da situação da vigilância sanitária no Brasil. No entanto, os achados permitem supor que os fatores facilitadores e as dificuldades identificadas na realização dessas ações específicas de saúde, numa metrópole com o porte de terceira maior cidade brasileira, são possivelmente semelhantes; se não na maioria, pelo menos em grande parte dos serviços de vigilância sanitária no país. 


\section{Colaboradores}

AA Bastos participou na concepção e elaboração do projeto, desenvolvimento dos instrumentos, preparação e execução da coleta de dados, montagem e análise do banco de dados e redação do artigo. EA Costa e LLC Castro participaram das fases de concepção e elaboração do projeto, preparação da coleta, desenvolvimento dos instrumentos e análise e redação do artigo.

\section{Referências}

1. Melo JEB. A indústria farmacêutica e a propaganda de medicamentos. In: Bonfim JRA, Mercucci VL, organizadores. A construção da política de medicamentos. São Paulo: Hucitec/Sobravime; 1997. p. 290292.

2. Sevalho G. O medicamento percebido como objeto híbrido: uma visão crítica do uso racional. In: Acurcio FA, organizador. Medicamentos e assistência farmacêutica. Belo Horizonte: Coopmed; 2003. p. 1-9.

3. Costa EA. Vigilância sanitária: proteção e defesa da saúde. São Paulo-Brasília: Sobravime; 2004.

4. Costa EA. Vigilância sanitária: proteção e defesa da saúde. In: Rouquayrol MZ, Almeida Filho N. Epidemiologia é Saúde. Rio de Janeiro: Medsi; 2003. p. 357-387.

5. Oshiro ML, Castro LLC. Avaliação dos efeitos de uma intervenção educativa para promoção do uso da Terapia de Reidratação Oral (TRO) em trabalhadores de farmácias. Cad Saude Publica 2002; 18(1):287-297.

6. Barros JAC. Nuevas tendencias de la medicalizacion. Cien Saude Colet 2008; 13(Supl.):579-587.

7. Castro LLC, Costa AM, Kozoroski AM, Rossini A, Cymrot R. Algumas características da prática da automedicação em Campo Grande, Mato Grosso do Sul. Revista de Ciências Farmacêuticas 2000; 21(1):81-101.

8. Edwards R. The accelerating need for pharmacovigilance. J R Coll Physicians Lond 2000; 34(1):48-51.

9. Melo MGM. A regulamentação sanitária e sua influência na definição do cenário farmacêutico do país. In: Acurcio FA, organizador. Medicamentos e assistência farmacêutica. Belo Horizonte: Coopmed; 2003. p. 65-72.

10. Netto AA, Mioto R. Automedicação tópica ocular: comercialização de colírios em farmácias. Rev Bras Oftalmol 2000; 59(2):108-114.

11. Camargo MDF, Montebelo MI, Lopes LC. Avaliação da adesão as normas da portaria 344/98/SVS/ MS, utilizando notificações e receitas oriundas da cidade de Salto/SP. Rev Ciênc Farm Básica Apl 2005; 26(2):131-137.

12. Vitor RS, Lopes CP, Menezes HS, Kerkhoff, CE. Padrão de consumo de medicamentos sem prescrição médica na cidade de Porto Alegre, RS. Cien Saude Colet 2008; 13(Supl.):737-743.

13. Volpato DE, Souza BV, Dalla Rosa LG, Melo LH, Daudt CAS, Deboni L. Use of antibiotics without medical prescription. Braz J Infect Dis 2005; 9(4):288291.

14. Lourenço ELB, Zubioli A, Baroni S, Cuman RKN, Silva MARCP, Assef SMC, Amado CAB. Consumo de antiinflamatórios esteroides em farmácia comunitária. Arquivos de Ciências da Saúde UNIPAR 2002: 6(3):93-96.

15. Bortolon PC, Medeiros EFF, Naves JOS, Karnikowski, MGO, Nóbrega OT. Análise do perfil de automedicação em mulheres idosas brasileiras. Cien Saude Colet 2008; 13(4):1219-1226. 
16. Ajalla MEA, Castro LLC. Contribuição para melhoria da prática farmacêutica no atendimento de pacientes com queixas referentes a doenças sexualmente transmissíveis, em Campo Grande-MS. Revista de Ciências Farmacêuticas 2003; 24(2):121-30.

17. Naves JOS. Orientação farmacêutica para DST nas farmácias do DF: um estudo de intervenção [tese]. Brasília: Univ. de Brasília; 2006.

18. Giacomini A, Kliemann DA, Tavares FF, Rodrigues GU, Coser PL. Aquisição de medicamentos em farmácias sem prescrição médica uma prática comum. Otite média aguda. Pediatria Moderna 2000; 36(4):177-181.

19. Silva RA, Marques FD, Goes PSA. Fatores associados à automedicação em dor de dente: análise a partir dos profissionais dos estabelecimentos farmacêuticos da cidade do Recife, PE. Cien Saude Colet 2008; 13(Supl.):697-701.

20. Capellá D, Laporte JR. Métodos aplicados em estúdios descriptivos de utilización de medicamentos. In: Laporte JR, Tognoni G, organizadores. Princípios de epidemiologia del medicamento. Barcelona: Salvat; 1993. p. 67-87.

21. Menezes CA, Magalhães SMS. Qualidade terapêutica de medicamentos adquiridos em drogarias da região central de Belo Horizonte-MG. Revista de Ciências Farmacêuticas 2004; 25(2):1449-1550.

22. Mendes Gonçalves RB. O processo tecnológico do trabalho em saúde. Divulg Saúde Debate 1991; 4:97102.

23. Mendes Gonçalves RB. Tecnologia e organização social das práticas de saúde: características tecnológicas de processo de trabalho na rede estadual de centros de saúde de São Paulo. São Paulo: Hucitec/Abrasco; 1994.

24. Scharaiber LB, Nemes MIB. Processo de trabalho e avaliação de serviços em saúde. Cadernos Fundap 1996; 19:106-21.

25. Merhy EE. Em busca do tempo perdido: a micorpolítica do trabalho vivo em saúde. In: Merhy EE, Onocko R. organizadores. Agir em saúde: um desafio para o público. São Paulo: Hucitec; 1997.

26. Merhy EE. O SUS e dos seus dilemas: mudar a gestão e a lógica do processo de trabalho em saúde (um ensaio sobre a micropolítica do trabalho vivo) In: Teixeira SMF, organizador. Saúde e democracia: a luta do Cebes. São Paulo; 1997.

27. Souza GS. Trabalho em vigilância sanitária: o controle sanitário da produção de medicamentos no Brasil [tese]. Salvador: Univ. Federal da Bahia; 2004.

28. Bruyne P, Herman J, Schoutheete M. Os modos de investigação. In: Bruyne P, Herman J, Schoutheete M, organizadores. Dinâmica da pesquisa em ciências sociais - os pólos da prática metodológica. Rio de Janeiro: Livraria Francisco Alves Editora S.A; 1977. p. 221-251

29. Yin RK. Estudo de caso: planejamento e métodos. Porto Alegre: Bookman; 2005.
30. Emerson RM, Fretz RI, Shaw LL. Processing fieldnotes: coding and memoing. In: Emerson RM, Fretz RI, Shaw LL. Writing ethnographic fieldnotes. Chicago and London: The University of Chicago Press; 1995. p. 142-68.

31. Minayo MCS. O desafio do conhecimento: pesquisa qualitativa em saúde. $8^{\mathrm{a}}$ ed. São Paulo-Rio de Janeiro: Hucitec-Abrasco; 2004.

32. Bardin L. Análise de Conteúdo. Lisboa: Edições 70; 1977.

33. Brasil. Lei no 5.991 de 17 de dezembro de 1973. Dispõe sobre o controle sanitário do comércio de drogas, medicamentos, insumos farmacêuticos e correlatos, e dá outras providências. Diário Oficial da União 1973; 19 dez.

34. Brasil. Lei no 6.360 de 23 de setembro de 1976. Dispõe sobre a vigilância sanitária a que ficam sujeitos os medicamentos, as drogas, os insumos farmacêuticos e correlatos, cosméticos, saneantes e outros produtos, e dá outras providências. Diário Oficial da União 1976; 24 set.

35. Brasil. Lei no 6.368 de 21 de outubro de 1976. Dispõe sobre medidas de prevenção e repressão ao tráfico ilícito e uso indevido de substâncias entorpecentes ou que determinem dependência física ou psíquica, e dá outras providências. Diário Oficial da União 1976; 22 out.

36. Brasil. Decreto $n^{\circ} .74 .170$ de 10 de junho de 1974 . Regulamenta a Lei $n^{\circ} .5 .991$ de 17 de dezembro de 1973 que dispõe sobre o controle sanitário do comércio de drogas, medicamentos, insumos farmacêuticos e correlatos. Diário Oficial da União 1977; 11 jun.

37. Brasil. Portaria n ${ }^{\circ} .802$ de 08 de outubro de 1998. Institui o Sistema de Controle e Fiscalização em toda a cadeia dos produtos farmacêuticos. Diário Oficial da União 1999; 07 abr.

38. Paim JS. Saúde, Política e reforma sanitária. Salvador: CEPS-ISC; 2002.

39. Teixeira CF. Planejamento e programação situacional em Distrito Sanitário: metodologia e organização. In: Mendes EV, organizador. Distrito Sanitário: processo social de mudança das práticas do SUS. São Paulo: Hucitec; 1993. p. 237-265.

40. Teixeira CF, Paim JS. Planejamento e programação de ações intersetoriais para a promoção da saúde e da qualidade de vida. Rev Adm Pública 2000; 34(6):63-80.

41. Shuqair NSMSAQ. A política de vigilância sanitária de medicamentos: um estudo de caso na região da Lapa - município de São Paulo [dissertação]. São Paulo: Univ. de São Paulo; 1996.

Artigo apresentado em 02/05/2008

Aprovado em 29/03/2009

Versão final aprovada em 20/04/2009 